\title{
Experimental implementation of the gyrator transform
}

\author{
José A. Rodrigo, * Tatiana Alieva, and María L. Calvo \\ Facultad de Ciencias Físicas, Universidad Complutense de Madrid, Ciudad Universitaria s/n, Madrid 28040, Spain \\ *Corresponding author: jarmar@fis.ucm.es
}

Received April 26, 2007; accepted June 15, 2007; posted June 20, 2007 (Doc. ID 82352); published September 12, 2007

\begin{abstract}
The gyrator transform (GT) promises to be a useful tool in image processing, holography, beam characterization, mode transformation, and quantum information. We introduce what we believe to be the first flexible optical experimental setup that performs the GT for a wide range of transformation parameters. The feasibility of the proposed scheme is demonstrated on the gyrator transformation of Hermite-Gaussian modes. For certain parameters the output mode corresponds to the Laguerre-Gaussian one. (C) 2007 Optical Society of America

OCIS codes: $070.2590,120.4820,200.4740,140.3300$.
\end{abstract}

The gyrator transform (GT) introduced in [1,2] and extensively studied in [3] as well as the fractional Fourier transform [4] belong to the class of the linear canonical integral transforms widely used for the optical, digital, and quantum information processing, holographic recording, mode transformation, etc. The GT at parameter $\alpha$, which we denote as a transformation angle, of a function $g_{i}\left(\mathbf{r}_{i}\right)$, is defined as

$$
\begin{aligned}
g_{o}\left(\mathbf{r}_{o}\right)= & R^{\alpha}\left[g_{i}\left(\mathbf{r}_{i}\right)\right]\left(\mathbf{r}_{o}\right)=\frac{1}{|\sin \alpha|} \iint g_{i}\left(x_{i}, y_{i}\right) \\
& \times \exp \left(i 2 \pi \frac{\left(x_{0} y_{o}+x_{i} y_{i}\right) \cos \alpha-\left(x_{i} y_{o}+x_{o} y_{i}\right)}{\sin \alpha}\right) \mathrm{d} x_{i} \mathrm{~d} y_{i},
\end{aligned}
$$

where $\mathbf{r}_{i, o}=\left(x_{i, o}, y_{i, o}\right)$ are the input and output coordinates, respectively. This transform is additive and periodic with respect to $\alpha$. For $\alpha=0$ it corresponds to the identity transform, for $\alpha=\pi / 2$ it reduces to the direct/inverse Fourier transform with rotation of the coordinates at $\pi / 2$, and for $\alpha=\pi$ the reverse transform described by the kernel $\delta\left(\mathbf{r}_{o}\right.$ $\left.+\mathbf{r}_{i}\right)$ is obtained. The applications of the GT for spacevariant filtering, hyperbolic noise reduction, and encryption have been proposed in [5]. Moreover the GT corresponds to the movement on the main meridian of the orbital Poincaré spheres [6,7] introduced by the analogy to the polarization Poincaré sphere. The GT can be considered a universal mode converter, since it allows the generation of all essentially different structurally stable Gaussian modes, which can be obtained from the Hermite-Gaussian (HG) modes by the integral canonical transforms [7].

To use the GT for optical information processing we need an optical setup performing this operation for different parameters $\alpha$. The design for such a system has been proposed in [9]. Based on the $A B C D$ matrix formalism for the first-order lossless optical systems, it has been shown that the coherent optical system, which contains three generalized lenses with fixed distances between them [Fig. 1(a)] is able to perform the GT for the large range of angles $\alpha$. The transformation angle $\alpha$ is changed by rotation of the cylindrical lenses, which forms the generalized lenses.

In this paper the first experimental implementation of the flexible optical scheme for the GT is reported. The action of this system is demonstrated on the example of the transformation of the $\mathrm{HG}$ modes into the helicoidal Laguerre-Gaussian (LG) ones for $\alpha=(2 k+1) \pi / 4$ ( $k$ is an integer) passing through intermediate modes $[7,8]$ for other values $\alpha$.

We start from a detailed description of the symmetric optical setup constructed with three generalized lenses performing the GT [Fig. 1(a)]. Every generalized lens is a combination of two convergent thin cylindrical lenses of the same power. The action of the generalized lens leads to the quadratic phase modulation written as

$$
g_{o}\left(x_{o}, y_{o}\right)=\exp \left(-i \pi \frac{x_{o}^{2}+y_{o}^{2}-2 x_{o} y_{o} \sin 2 \varphi}{\lambda f}\right) g_{i}\left(x_{o}, y_{o}\right)
$$

where $\lambda$ is the wavelength, $f$ is the focal distance of a cylindrical lens, and angle $\varphi$ indicates the position of the axis of symmetry of the cylindrical lenses. Thus the axis of the cylindrical lenses forms angle $\phi_{1}=-\varphi$ and $\phi_{2}$ $=\varphi-\pi / 2$ with the vertical axis $O Y$, respectively $[10,11]$ [see Fig. 1(b)]. The first and third generalized lenses are identical and will be further denoted as $\mathbf{L}_{1}$. Their focal distance $f_{1}$ equals the distance $z$ between two consecutive generalized lenses of the setup (the first and second or the second and third generalized lenses [Fig. 1(a)]. The second generalized lens $\mathbf{L}_{2}$ has a focal distance $f_{2}=z / 2$. As an example, the phase modulation functions associated to each of the generalized lenses for the GT at angle $\alpha$ $=3 \pi / 4 \quad(z=0.5 \mathrm{~m}$ and $\lambda=532 \mathrm{~nm})$ are also shown in Fig. 1(a). 


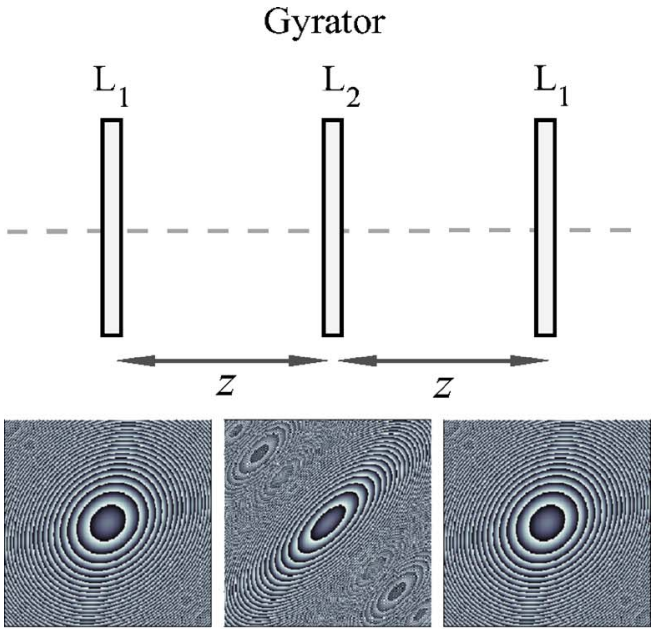

(a)

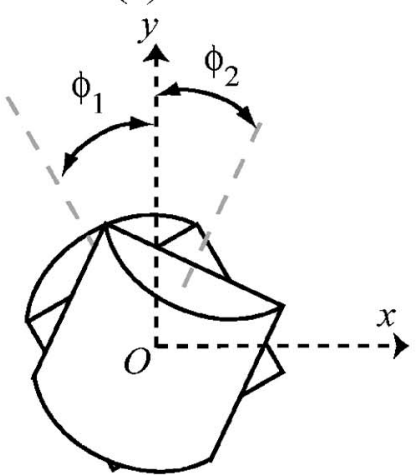

(b)

Fig. 1. (Color online) (a) Setup scheme associated with the gyrator transform. The phase modulation functions (for the case $z$ $=0.5 \mathrm{~m}$ and $\lambda=532 \mathrm{~nm}$ ) associated with each generalized lens when the setup performs the GT at angle $\alpha=3 \pi / 4$ are shown below, where the gray levels indicate the phase distribution range $[-\pi, \pi]$. (b) An assembled set of two cylindrical lenses that forms the generalized lenses $\mathbf{L}_{1}$ and $\mathbf{L}_{2}$.

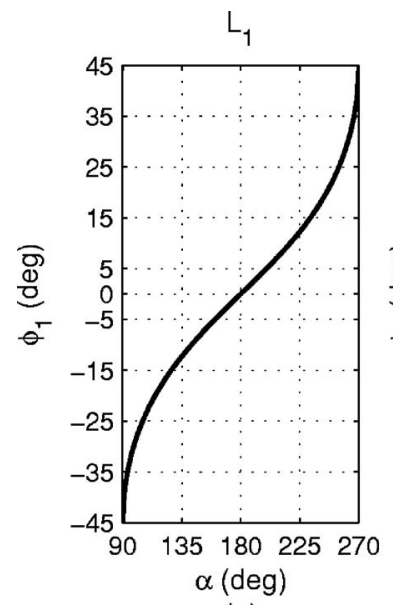

(a)

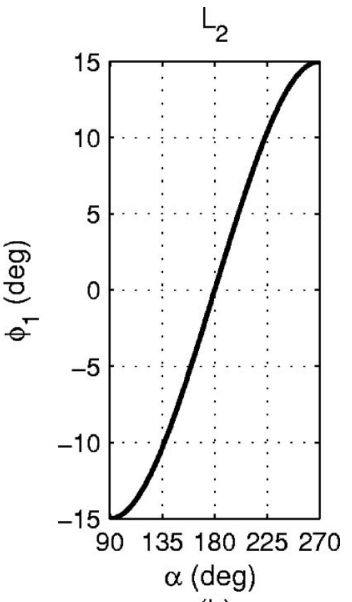

(b)
Fig. 2. Operation curves $\phi_{1}(\alpha)$ for the generalized lenses (a) $\mathbf{L}_{1}$ and (b) $\mathbf{L}_{2}$. The operation curve $\phi_{2}(\alpha)$ is derived from the relation $\phi_{2}=-\left(\phi_{1}+\pi / 2\right)$.

Let us consider the evolution of the complex field $g_{i}\left(x_{i}, y_{i}\right)$ during the propagation through this system. Using the Fresnel diffraction integral and Eq. (2) for lens $\mathbf{L}_{1}$ we obtain the expression for the complex field just before the second lens $\mathbf{L}_{2}$,

$$
\begin{aligned}
g_{1}\left(x_{1}, y_{1}\right)= & \frac{1}{i \lambda z} \exp \left(i \pi \frac{x_{1}^{2}+y_{1}^{2}}{\lambda z}\right) \iint g_{i}\left(x_{i}, y_{i}\right) \\
& \times \exp \left(-i 2 \pi \frac{x_{1} x_{i}+y_{1} y_{i}-x_{i} y_{i} \sin 2 \varphi_{1}}{\lambda z}\right) \mathrm{d} x_{i} \mathrm{~d} y_{i} .
\end{aligned}
$$

After the corresponding modulation by the second lens transfer function and the propagation through a freespace interval $z$ we derive

$$
g_{2}\left(x_{2}, y_{2}\right)=-\frac{1}{\left|2 \lambda z \sin 2 \varphi_{2}\right|} \exp \left(i \pi \frac{x_{2}^{2}+y_{2}^{2}}{\lambda z}\right) \iint g_{i}\left(x_{i}, y_{i}\right) \exp \left(i \pi \frac{2 x_{i} y_{i} \sin 2 \varphi_{1}-\left(x_{2} y_{i}+x_{i} y_{2}+x_{i} y_{i}+x_{2} y_{2}\right) / \sin 2 \varphi_{2}}{\lambda z}\right) \mathrm{d} x_{i} \mathrm{~d} y_{i}
$$

The action of the third lens, $\mathbf{L}_{1}$, leads to the final expression for the complex field amplitude at the output plane of the gyrator system:

$$
g_{o}\left(x_{o}, y_{o}\right)=-\frac{1}{\left|2 \lambda z \sin 2 \varphi_{2}\right|} \iint g_{i}\left(x_{i}, y_{i}\right) \exp \left(i 2 \pi \frac{\left(x_{o} y_{o}+x_{i} y_{i}\right)\left(2 \sin 2 \varphi_{1} \sin 2 \varphi_{2}-1\right)-\left(x_{o} y_{i}+x_{i} y_{o}\right)}{2 \lambda z \sin 2 \varphi_{2}}\right) \mathrm{d} x_{i} \mathrm{~d} y_{i} .
$$

This equation coincides, except for a constant phase, with the definition of the gyrator transform at angle $\alpha$ with normalization $s^{2}=\lambda z$, Eq. (1), if

$$
\begin{aligned}
& \sin 2 \varphi_{1}=\cot (\alpha / 2), \\
& \sin 2 \varphi_{2}=(\sin \alpha) / 2 .
\end{aligned}
$$

Note that the angles $\varphi_{1}$ and $\varphi_{2}$ correspond to the generalized lenses $\mathbf{L}_{1}$ and $\mathbf{L}_{2}$, respectively. It is easy to see from the later relation that this setup is able to perform the GT only for the angles $\alpha \in[\pi / 2,3 \pi / 2]$. Nevertheless, since $R^{\alpha+\pi}\left[f_{i}\left(\mathbf{r}_{i}\right)\right]\left(\mathbf{r}_{o}\right)=R^{\alpha}\left[f_{i}\left(\mathbf{r}_{i}\right)\right]\left(-\mathbf{r}_{o}\right)$ we can cover all the interval $\alpha \in[0,2 \pi]$ if it is necessary.

The variation of the transformation angle $\alpha$ is achieved by proper rotation of the cylindrical lenses according to the operation curves. The operation curve is a graphical representation of the rotation angle $\phi_{1}$ of one of the cylindrical lenses, which composed a given generalized lens as 


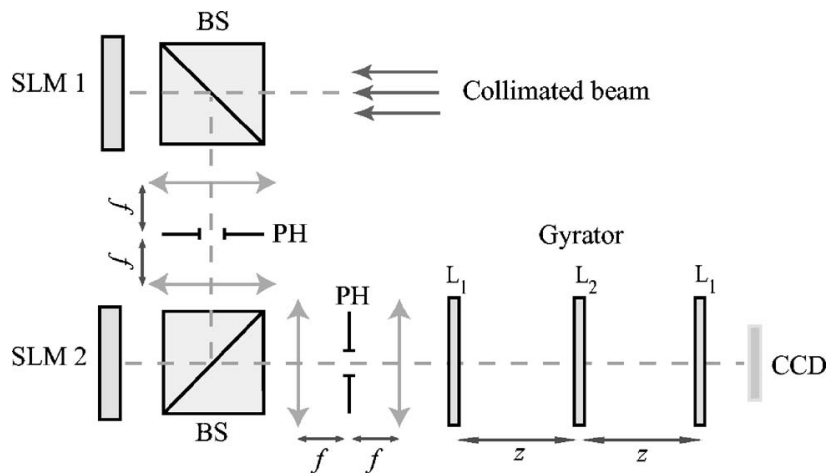

Fig. 3. Experimental optical system configuration. Two SLMs (Holoeye LCR-2500) are used for the input signal (HG mode) generation. BS is a beam splitter. A pinhole $(\mathrm{PH})$ is placed at the Fourier plane of the $4-f$ system. The GT at angle $\alpha$ is performed by three generalized lenses. The parameter $\alpha$ is changed by the proper rotation of the cylindrical lenses, which form every generalized lens. The output signal is registered using a CCD camera (Sony XCD-X710).

a function of the transformation angle $\alpha$. In Fig. 2 the operation curves $\phi_{1}(\alpha)$ for the generalized lens $\mathbf{L}_{1}(\mathrm{a})$ and $\mathbf{L}_{2}$ (b) are shown. Note that $\phi_{2}(\alpha)$ is derived from the relation $\phi_{2}=-\left(\phi_{1}+\pi / 2\right)$. In addition, when the angle between the cylindrical lenses is $\pi / 2$ a generalized lens reduces to a spherical one.

We observe that for $\alpha=\pi$, occurring when the generalized lenses $\mathbf{L}_{1}$ and $\mathbf{L}_{2}$ are reduced to the spherical lenses, the common 4- $f$ system-a cascade of two Fourier transforming systems-is obtained. Therefore the proposed gyrator system can be considered as a generalization of the well-known optical processing $4-f$ system to the case of the generalized lenses.

In Fig. 3 the detailed scheme of the GT optical setup is displayed. The input complex field amplitude is generated by the two coupled spatial light modulators (SLMs). This optical configuration is known as a hybrid hologram [12] because the amplitude and phase components of the input signal are separately implemented on each SLM. The amplitude component is projected by means of a $4-f$ lens system on the second SLM, which introduces the appropriate phase modulation. The second $4-f$ lens system is used in order to remove the nonzero diffraction orders in the Fourier plane, arising due to the discrete structure of the SLMs. The main disadvantage of this hybrid hologram scheme concerns the alignment accuracy between the SLM displays. Nevertheless, position stages for the SLMs are not required because the alignment between amplitude and phase components is digitally achieved by means of a PC. Therefore this alignment accuracy is limited by the pixel size, which is $20 \mu \mathrm{m}$ in our case (Holoeye LCR-2500 SLM). The signal quality is also limited by the spatial resolution and in addition by the dynamic range of the SLM ( 8 bits). The application of the SLMs for an input signal generation allows performance of an almost real time information processing. The distance between generalized lenses in our experimental setup is $z=0.5 \mathrm{~m}$.

For the demonstration of the experimental implementation of the GT we use the HG modes of various orders as input signals. The HG mode is given by the following expression:

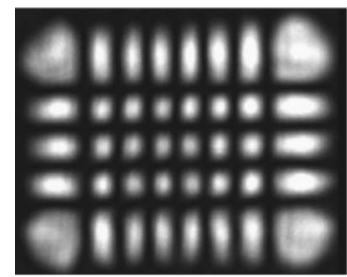

(a)

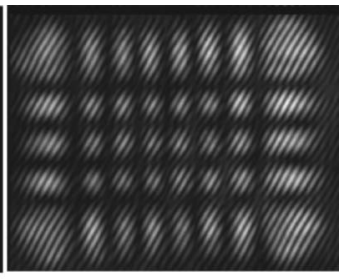

(b)
Fig. 4. Hermite-Gaussian mode $\mathrm{HG}_{7,4}$ obatined using the proposed hybrid hologram. The image (a) corresponds to $\mathrm{HG}_{7,4}$ intensity distribution and (b) is an interference pattern, which reveals the $\mathrm{HG}_{7,4}$ phase distribution.

$$
\mathrm{HG}_{m, n}(\mathbf{r} ; w)=2^{1 / 2} \frac{H_{m}\left(\sqrt{2 \pi} \frac{x}{w}\right) H_{n}\left(\sqrt{2 \pi} \frac{y}{w}\right)}{\sqrt{2^{m} m ! w} \sqrt{2^{n} n ! w}} \exp \left(-\frac{\pi}{w^{2}} \mathbf{r}^{2}\right),
$$

where $H_{m}$ is the Hermite polynomial and $w$ is the beam waist. The beam waist is set at $w=0.73 \mathrm{~mm}$ for the HG modes considered in this work. The HG modes $\mathrm{HG}_{m, n}(\mathbf{r} ; w)$ generated experimentally by the modulation of the collimated Nd:YAG laser beam $(\lambda=532 \mathrm{~nm})$ by the coupled SLMs with further spatial filtering of the nonzero order diffraction components are in good agreement with the theoretical results. As an example, the intensity distribution (a) of the $\mathrm{HG}_{7,4}$ mode and its phase structure in the form of the interferrogram with a plane wave (b) are demonstrated in Fig. 4. The images are registered by CCD camera, Sony XCD-X710.

The rest of the results shown in Figs. 5-7 are related to the functioning of the gyrator transform setup. It is
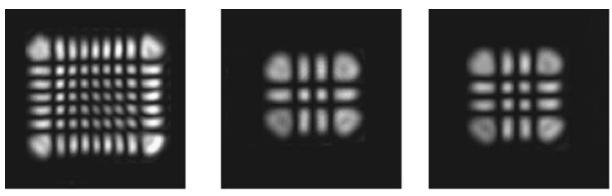

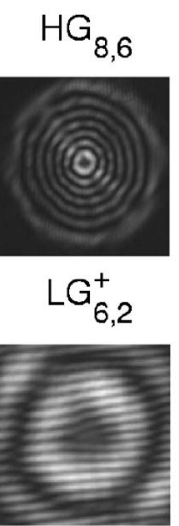

(a)

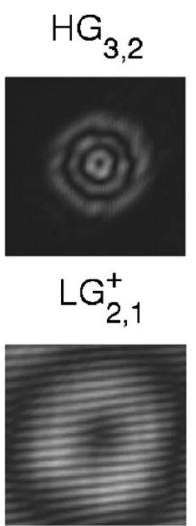

(b)
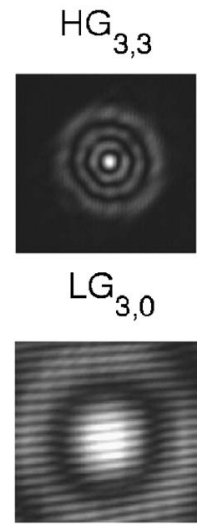

(c)
Fig. 5. Input $\mathrm{HG}$ modes $\mathrm{HG}_{8,6}, \mathrm{HG}_{3,2}$, and $\mathrm{HG}_{3,3}$ are transformed by the GT at $\alpha=3 \pi / 4$ into $\mathrm{LG}$ modes $\mathrm{LG}_{6,2}^{+}, \mathrm{LG}_{2,1}^{+}$, and $\mathrm{LG}_{3,0}$, respectively. The interferrograms for these LG modes with a plane wave are displayed in (a)-(c), correspondingly. Images (a) and (b) reveal a forklike structure, typical for the associated helicoidal phase distribution, which is not present for the case of (c) $\mathrm{LG}_{3,0}$. 


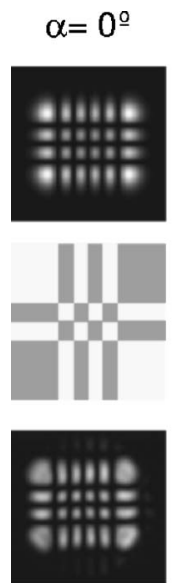

(a)
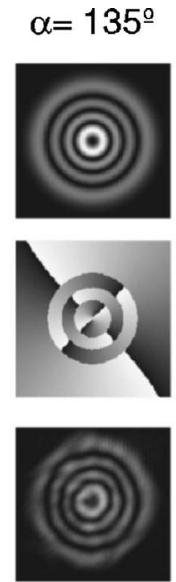

(b)

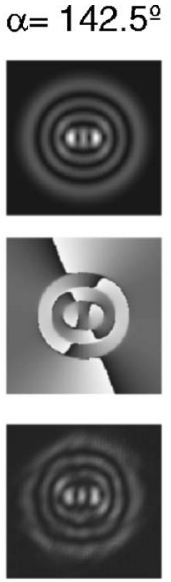

(c)
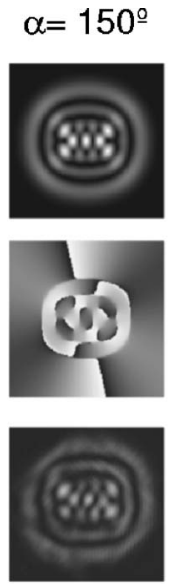

(d)

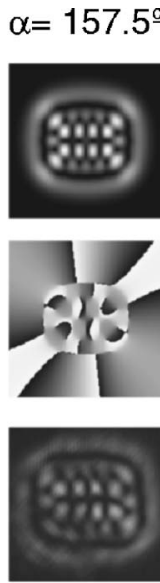

(e) $\alpha=180^{\circ}$
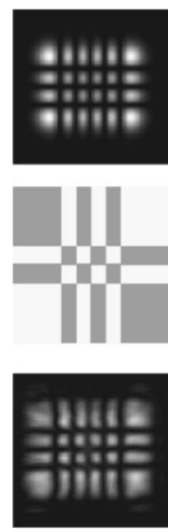

(f)

Fig. 6. Intermediate modes obtained by the GT of the input mode $\mathrm{HG}_{5,3}$ (a) for the angles $\alpha=[3 \pi / 4, \pi]$, (b)-(f). The first and second rows correspond to the intensity and phase distributions (gray levels indicate the phase range $[-\pi, \pi]$ ), respectively, obtained by numerical simulations of the GT. The experimental intensity distributions are shown in the third row. The input mode $(\alpha=0)$ generated by the SLMs is also displayed in the third row, (a).

known that by the GT at angles $\alpha=(2 k+1) \pi / 4(k$ is an integer) the $\mathrm{HG}$ modes $\mathrm{HG}_{m, n}(\mathbf{r} ; w)$ are transformed into the helicoidal LG modes:

$$
\begin{aligned}
\mathrm{LG}_{p, l}^{ \pm}(\mathbf{r} ; w)= & w^{-1} \sqrt{\frac{\min (m, n) !}{\max (m, n) !}}\left(\sqrt{2 \pi}\left(\frac{x}{w} \pm i \frac{y}{w}\right)\right)^{l} \\
& \times L_{p}^{l}\left(\frac{2 \pi}{w^{2}} \mathbf{r}^{2}\right) \exp \left(-\frac{\pi}{w^{2}} \mathbf{r}^{2}\right)
\end{aligned}
$$

where $L_{p}^{l}$ is the Laguerre polynomial, $p=\min (m, n)$ and $l$ $=|m-n|$. The topological charge, related to the orbital angular momentum (OAM) of the vortex mode, is given by $\pm l$. In particular for the angle $\alpha=3 \pi / 4$ the $\mathrm{HG}_{8,6}, \mathrm{HG}_{3,2}$, and $\mathrm{HG}_{3,3}$ modes are transformed into $\mathrm{LG}_{6,2}^{+}, \mathrm{LG}_{2,1}^{+}$, and $\mathrm{LG}_{3,0}$. The experimental results of this transformation are shown in Fig. 5. The interference pattern of the LG mode with a plane wave reveals its helicoidal phase structure, as displayed in Figs. 5(a)-5(c).

For $\alpha=\pi k / 2$ the output mode corresponds to $\mathrm{HG}_{m, n}$ rotated at angle $\alpha$ with an additional phase shift.

For the rest of the transformation angles the intermediate modes (or gyrating modes [7]), which possess the fractional orbital angular momentum [6], are obtained. In particular in Fig. 6 the modes generated from the input mode $\mathrm{HG}_{5,3}$ [Fig. 6(a)] by the GT for the angles $\alpha$ $\in[3 \pi / 4, \pi]$ are displayed. The first and the second rows show the numerical simulation of the intensity and phase

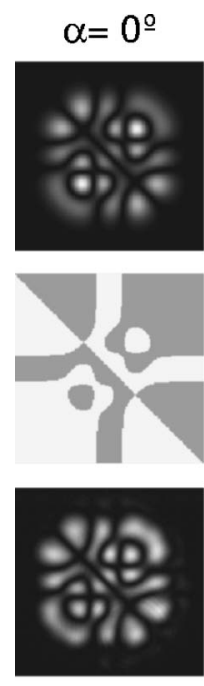

(a)
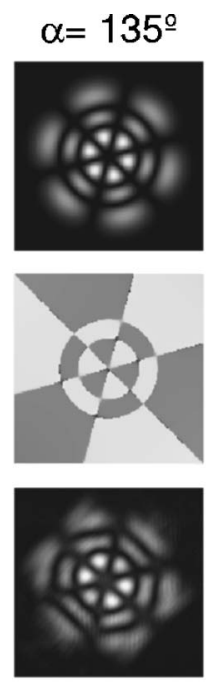

(b)
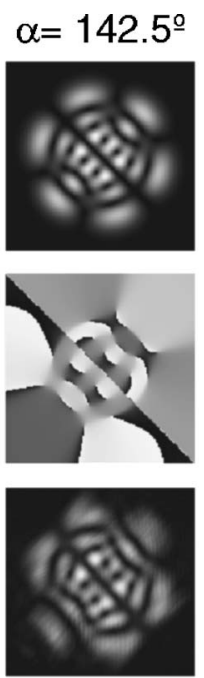

(c)
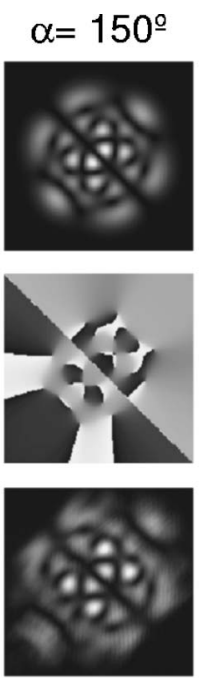

(d)
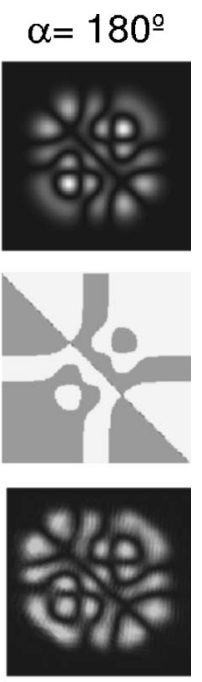

(e)

Fig. 7. Intermediate modes obtained by the GT of the $\mathrm{HG}$ modes composition $\mathrm{HG}_{5,2}+\mathrm{HG}_{2,5}$ (a) for the angles $\alpha=[3 \pi / 4, \pi]$, (b)-(e). The first and second rows correspond to the intensity and phase distributions (gray levels indicate the phase range $[-\pi, \pi]$ ), respectively, obtained by numerical simulations of the GT. The experimental intensity distributions are shown in the third row. The input mode $(\alpha$ $=0$ ) generated by the SLMs is also displayed in the third row, (a). 
distributions of these modes, respectively. The third row in Figs. 6(a)-6(f) corresponds to the experimental intensity distribution registered by means of the CCD camera. The continued gyrator transformation of the HG mode $\mathrm{HG}_{m, n}$ for the angles $\alpha \in[\pi / 2,3 \pi / 2]$ is related to the movements along the main meridian of the orbital $(m, n)$ Poincaré sphere [6,7].

Moreover, a composition of the HG modes of the same order $(n+m=$ const $)$ also produces a structurally stable mode under the GT action. For instance the combination of $\mathrm{HG}_{5,2}$ and $\mathrm{HG}_{2,5}$ modes: $\mathrm{HG}_{5,2}+\mathrm{HG}_{2,5}$ [Fig. 7(a)] leads for $\alpha=3 \pi / 4$ to the odd LG mode, which is the sum of two helicoidal LG modes with opposite OAM values: $\mathrm{LG}_{2,3}^{+}$ $+\mathrm{LG}_{2,3}^{-}$[Fig. 7(b)]. The intermediate modes are displayed in Figs. 7(c) and 7(e). We notice that due to the input mode symmetry it is an eigenfunction for GT at the angle $\alpha=\pi$, which is a simple $\pi$-angle rotation [see Fig. 7(e)]. The first and second rows again show the numerical simulation of the intensity and phase distributions of these modes. Meanwhile the third row [Figs. 7(a)-7(e)] corresponds to the experimental intensity distribution.

We observe that the experimental results are in excellent agreement with the theoretical predictions that demonstrate the feasibility of the proposed GT setup. The flexible structure of this scheme based on the fixed distances between the three assembled pairs of the cylindrical lenses and the manipulation of the transformation angle $\alpha$ by means of the lens rotation makes the setup useful for numerous applications. In particular the proposed experimental setup is able to generate, almost in real time, a wide spectrum of modes applicable for beam design purposes, optical trapping, quantum information, etc. Moreover, regarding the promising results related to the application of the GT for shift-variant filtering, noise reduction, and encryption [5], this setup can be used for optical image processing.

\section{ACKNOWLEDGMENTS}

The Spanish Ministry of Education and Science is acknowledged for financial support, project TEC 200502180/MIC.

\section{REFERENCES}

1. R. Simon and K. B. Wolf, "Structure of the set of paraxial optical systems," J. Opt. Soc. Am. A 17, 342-355 (2000).

2. K. B. Wolf, Geometric Optics on Phase Space (SpringerVerlag, 2004).

3. J. A. Rodrigo, T. Alieva, and M. L. Calvo, "Gyrator transform: properties and applications," Opt. Express 15, 2190-2203 (2007).

4. H. M. Ozaktas, Z. Zalevsky, and M. Alper Kutay, The Fractional Fourier Transform with Applications in Optics and Signal Processing (Wiley, 2001).

5. J. A. Rodrigo, T. Alieva, and M. L. Calvo, "Gyrator transform for image processing," Opt. Commun., in press, doi: 10.1016/j.optcom.2007.06.023.

6. G. F. Calvo, "Wigner representation and geometric transformations of optical orbital angular momentum spatial modes," Opt. Lett. 30, 1207-1209 (2005).

7. T. Alieva and M. Bastiaans, "Orthonormal mode sets for the two-dimensional fractional Fourier transformation," Opt. Lett. 32, 1226-1228 (2007).

8. E. G. Abramochkin and V. G. Volostnikov, "Generalized Gaussian beams,” J. Opt. A, Pure Appl. Opt. 6, S157-S161 (2004).

9. J. A. Rodrigo, T. Alieva, and M. L. Calvo, "Optical system design for ortho-symplectic transformations in phase space," J. Opt. Soc. Am. A 23, 2494-2500 (2006).

10. J. Shamir, "Cylindrical lens described by operator algebra," Appl. Opt. 18, 4195-4202 (1979).

11. G. Nemes and A. E. Seigman, "Measurement of all ten second-order moments of an astigmatic beam by use of rotating simple astigmatic (anamorphic) optics," J. Opt. Soc. Am. A 11, 2257-2264 (1994).

12. V. A. Soifer, ed., Methods for Computer Design of Diffractive Optical Elements (Wiley, 2002). 